\title{
Modelling and solving of the allocation problem of non-convex polygons with rotations
}

\author{
Yu.G. Stoyan, M.V. Novozhilova \\ National Ukrainian Academy of Sciences \\ Institute for Problems in Machinery \\ 2/10, Pozharsky Str., Kharkov-46, 310046, Ukraine. \\ Tel: (0572) 9595 36. Fax: (0572) 942914. \\ e-mail: stoyan@ipmach .kharkov.ua
}

\begin{abstract}
The work deals with the problem of optimal allocation of objects of the so-called irregular form. The objects are allocated on a strip of given width. The rotation of objects are allowed.
\end{abstract}

Allocation, nonlinear optimization

\section{Keywords}

\section{INTRODUCTION}

The problem is insufficiently studied, but it is of great applied importance and also of interest for the developing the theory of solving cutting and packing problems, as the problem in question belongs just that very set of problems. Problems of cutting and packing are frequently found in different spheres of human activity (cutting glass, metal, textile, leather etc., allocation of equipment in complex shape spaces, design of complex engineering systems).

A broad range of bibliography (see Sweeney and Ridenour (1989)) in certain directions of research exists. It is necessary to mention the well-known works of Dowsland (1992), Beasley (1985), Terno (1993), Li and Milenkovic (1993), Schwartz and Sharir (1983). However, very few works can practically be found in which the allocation problems are investigated taking into account the rotation possibility of allocation objects or the allocation region. Apparently it is caused by their complexity and non-triviality of mathematical models, necessary for their adequate description.

A wide class of problems connected with allocation of $2 \mathrm{D}$ and 3D complex shape geometric objects in domains of arbitrary shape has been studied for a number of years at the Institute for problems in Machinery (Ukraine, Kharkov). 
In the work a mathematical model of the allocation problem being considered is constructed based on the original theory of $\Phi-$ functions and structures of nonlinear inequalities. The exact method searching for a problem local minimum from any feasible initial point uses the active set strategy ideas.

\section{PROBLEM MATHEMATICAL MODEL CONSTRUCTION}

Let there be in an arithmetic Euclidean space $R^{2}$ a connected region $T_{0}$ with a linear - piecewise frontier: $\left\{(x, y) \mid(x, y) \in R^{2}, 0 \leq y \leq A, 0 \leq x \leq z ; A\right.$ - const, $z-v a r\}$, given in a fixed orthogonal coordinate system $X O Y$. There also are objects $T_{i} \subset R^{2}, i=1,2, \ldots, n(n>1)$, which are canonically closed bounded point sets with an arcwise frontier and a connected interior (int). It is required to allocate objects $T_{i}$ without overlapping in region $T_{0}$ so that value $z$ reaches its minimum. In other words, it is necessary to determine

$\min z$

s.t. : $\quad T_{i} \subset T_{0}$

$\operatorname{int} T_{i} \cap \operatorname{int} T_{j}=\emptyset, i, j=1,2, \ldots, n, i \neq j$

where $\emptyset$ is empty set.

In general the allocation objects are non-convex polygons $T_{i}$ with allocation parameters $u_{i}=\left(v_{i}, \varphi_{i}\right)$, where $v_{i}=\left(x_{i}, y_{i}\right), v_{i} \in i n t T_{i}$ are coordinates of the origin of eigen coordinate system $O X_{i} Y_{i}, \varphi_{i}$ is angular parameter, which equals a rotation angle of the system $O X_{i} Y_{i}$ with respect to the system $O X Y$.

Allocation parameters $\left(v_{i}, \varphi_{i}\right)$ and variable $z$ induce space $R^{3 n+1}$ of allocation parameters, in which the problem (1-3) ought to be considered, and the conditions (2-3) describe the feasible region $D \subset R^{3 n+1}$ of the problem.

The initial information about allocation objects is an ordered set of vertices $\left\{t_{i}^{k}\left(x_{i}^{k}, y_{i}^{k}\right)\right\}$, $k=1, . ., N_{i}$ of object $T_{i}$ given in the system $O X_{i} Y_{i}$.

\section{$2.1 \Phi_{i j}\left(u_{i}, u_{j}\right)$-functions}

The constructive tool of formalizing the conditions of mutual non-overlapping of objects (condition (3)) is $\Phi_{i j}\left(u_{i}, u_{j}\right)$-functions introduced for each pair $(i, j)$ of objects being allocated (Stoyan (1980)). Moreover, the $\Phi_{0 j}\left(u_{i}, u_{j}\right)$-function can be also applied to describe the condition (2) of belonging of object $T_{i}$ to region $T_{0}$.

Definition 1 Any, everywhere defined and continuous function in $R^{6}$, possessing the following characteristic property:

$$
\begin{aligned}
& \Phi_{i j}\left(u_{i}, u_{j}\right)>0, \text { if } c l T_{i}\left(u_{i}\right) \bigcap c l T_{j}\left(u_{j}\right)=\emptyset, \\
& \Phi_{i j}\left(u_{i}, u_{j}\right)=0, \text { if } \begin{array}{c}
c l T_{i}\left(u_{i}\right) \bigcap \operatorname{cl} T_{j}\left(u_{j}\right) \neq \emptyset, \\
\text { int } T_{i}\left(u_{i}\right) \bigcap i n t T_{j}\left(u_{j}\right)=\emptyset,
\end{array}
\end{aligned}
$$


$\Phi_{i j}\left(u_{i}, u_{j}\right)<0$, if $\operatorname{int} T_{i}\left(u_{i}\right) \bigcap \operatorname{int} T_{j}\left(u_{j}\right) \neq \emptyset$

where clT is topological closure of set $T$, is called $\Phi$-function.

Hence, the characteristic property of the $\Phi_{i j}$-function allows to speak about nonintersection, tangency and intersection of corresponding point sets.

Later on we shall be interested in following properties of $\Phi_{0 j}\left(u_{i}, u_{j}\right)$. We assume that all the $\varphi_{i}=$ const.

Property 1 For objects $T_{i}$ and $T_{j}$ the surface of the $\Phi_{i j}\left(v_{i}, v_{j}\right)$-function defined by equation $\Phi_{i j}\left(v_{i}, v_{j}\right)=0$, is linear-piecewise.

Property 2 The region of non-negative values of the $\Phi_{i j}\left(v_{i}, v_{j}\right)$-function is a polyhedral closed set $D_{i j} \subset R^{4}$.

Property 3 The region $D_{i j} \subset R^{4}$ may be presented in the form $D_{i j}=\bigcup_{g=1}^{G_{i j}} \operatorname{Tr}^{g}, G_{i j} \leq$ $m_{i j} \leq O\left(K_{i}+K_{j}\right)$, where $\operatorname{Tr}^{g}$ is truncation, which is described by intersection of a finite number $P_{g}$ closed half-spaces $H^{l}, l \in\left\{1,2, \ldots, m_{i j}\right\}$, defined by generating planes $E^{l}$, i.e. $D_{i j}=\bigcup_{g=1}^{G_{i j}} \bigcap_{p=1}^{P g} H^{l}$, where $l=f(p, g)$.

The generating planes $E^{l}$ are defined by equations $f^{l}\left(v_{j}, v_{i}\right)=0$. Functions $f^{l}\left(v_{j}, v_{i}\right)$ have the form

$f^{l}\left(v_{j}, v_{i}\right)=a^{l} \cdot\left(x_{j}-x_{i}\right)+b^{l} \cdot\left(y_{j}-y_{i}\right)+c^{l}, l=1,2, \ldots, m_{i j}$,

where coefficients $a^{l}, b^{l}, c^{l}$ are functions of certain vertices of objects $T_{i}$ and $T_{j}$.

The position of point $\left(v_{j}, v_{i}\right)$ in half-space $H^{l}$ is determined by inequality $f^{l}\left(v_{j}, v_{i}\right) \leq 0$.

Let us consider two-valued predicate $S\left(f^{\prime}\left(v_{i}, v_{j}\right)\right)$, as Rvachev (1967), such that

$S\left(f^{l}\left(v_{i}, v_{j}\right)\right)= \begin{cases}1, \text { if } & f^{l}\left(v_{i}, v_{j}\right) \leq 0 \\ 0, \text { if } & f^{l}\left(v_{i}, v_{j}\right)>0\end{cases}$

Then predicate $\Omega_{D_{i j}}\left(v_{i}, v_{j}\right)$, characterizing region $D_{i j}$, may be presented in the following form:

$\Omega_{D_{i j}}\left(v_{i}, v_{j}\right)=\bigvee_{g=1}^{G_{i j}}\left(\bigwedge_{p=1}^{P_{g}} S\left(f_{l}\left(v_{i}, v_{j}\right)\right)\right), l \in\left\{1,2, \ldots, m_{i j}\right\}$

i.e. $\Omega_{D_{i j}}\left(v_{i}, v_{j}\right)=1$, if $\left(v_{i}, v_{j}\right) \in D_{i j}$, and $\Omega\left(v_{i}, v_{j}\right)=0$, if $\left(v_{i}, v_{j}\right) \notin D_{i j}$. 


\section{Structures of inequalities}

To describe region $D_{i j}$, defined by predicate $\Omega_{D_{i j}}$, we shall use the structure of linear inequalities, following by Magas (1984). To simplify the exposition, we shall say that the $l$-th and $r$-th inequalities of the form (7) are connected by the conjunction operation, if $S\left(f^{l}\right)$ and $S\left(f^{r}\right)$ enter together at least into the one conjunct in expression (9). Otherwise, we shall say that the $l$-th and the $r$-th inequalities are connected by the disjunction operation.

Definition 2 The structure of linear inequalities $S(F(x), \Delta, m)$ is an ordered set $F(x)$ of linear inequalities of the form $f_{t}(x) \geq 0\left(x \in R^{k}, t=1,2, \ldots, m\right)$ with defined operations of conjunction or disjunction for each pair of inequalities; the operations are determined by the relation matrix $\Delta=\left\|\delta_{l r}\right\|_{m \times m}$ such that $\delta_{l r}=1$ corresponds to the conjunction operation between the $l$-th and $r$-th inequalities $(l, r \in\{1,2, \ldots, m\})$ and $\delta_{l r}=0$ corresponds to the disjunction operation.

As is obvious from the definition, the notion of a structure of inequalities generalizes the notion of a system of inequalities. That is, if all the elements of matrix $\Delta$ of the inequalities structure $S(F(x), \Delta, m)$ are equal to unit, then the inequalities structure shall be identical to a system of inequalities of set $F(x)$. We denote the matrix of operations with unit elements as $\Delta^{1}$. Otherwise, if none pair of inequalities of structure $S(F(x), \Delta, m)$ is connected by conjunction operation, e.g. elements $\delta_{l l}=1$ only, then we denote such an operation matrix as $\Delta^{0}$.

Therefore to describe region $D_{i j}$ we shall use the structure $S_{g}\left(F_{i j}\left(v_{i}, v_{j}\right), \Delta, m_{i j}\right)$,

Then under assumption that all the $\varphi_{i}=$ const the conditions of mutual pairwise non-overlapping of $n$ objects being allocated are described by the structure

$$
\bigcap_{g=1}^{C_{2}^{n}} S_{g=y(i, j)}\left(F_{i j}\left(v_{i}, v_{j}\right), \Delta, m_{i j}\right),
$$

Using such a methodology one can define the analytical description of the allocation conditions of $n$ objects $T_{i}$ into region $T_{0}$ as the system

$$
\bigcap_{i=1}^{n} S_{i 0}\left(F_{i 0}\left(v_{i}, z\right), \Delta^{1}, m_{i 0}\right),
$$

where each system $S_{i 0}$ is a system of a four linear inequalities of the form:

$$
\begin{gathered}
x_{i}-z \leq-p_{i}^{l}, p_{i}^{l}=\max _{k} x_{i}^{k} ; \quad-x_{i} \leq p_{i}^{r}, p_{i}^{r}=\min _{k} x_{i}^{k} \\
y_{i} \leq-p_{i}^{d}, p_{i}^{d}=\min _{k} y_{i}^{k} ; \quad y_{i}+A \leq-p_{i}^{u}, p_{i}^{u}=\max _{k} x_{i}^{k}, \\
k=1, \ldots, N_{i},
\end{gathered}
$$

In the case if $\varphi_{i}$ are not const an ordered set $F(x)$ of non-linear inequalities with defined operations by analogy with above operations for each pair of inequalities is a structure of non-linear inequalities. 
The feasible region $D$ of the problem (1-3) is determined by the structure of non-linear inequalities.

\subsection{Analytical model of the basic problem}

Let set $C_{i}=\operatorname{conv} T_{i}$ be a convex bull of object $T_{i}$ and $m_{i}^{c}$ is the number of vertices of $C_{i}$. Then the structure $\Sigma_{0}$ of inequalities describing the object $T_{i}$ position in $T_{0}$ must include $4 \times m_{i}^{c}$ systems consisting of both support function $f_{c}\left(v_{i}, \varphi_{i}\right)$ of kind

$x_{i}-z \leq-x_{i}^{c}\left(\varphi_{i}\right), \quad y_{i} \leq-y_{i}^{c}\left(\varphi_{i}\right), \quad y_{i}+A \leq-y_{i}^{c}\left(\varphi_{i}\right), \quad-x_{i} \leq x_{i}^{c}\left(\varphi_{i}\right)$,

where $x_{i}^{c}\left(\varphi_{i}\right)=x_{i}^{c} \cos \varphi_{i}-y_{i}^{c} \sin \varphi_{i}, x_{i}^{c}\left(\varphi_{i}\right)=y_{i}^{c} \cos \varphi_{i}+x_{i}^{c} \sin \varphi_{i}$, and pair of inequalities which determine the range of parameter $\varphi_{i}$. Then structure $\Sigma_{0}$ has a form:

$$
\begin{aligned}
& S_{1}\left(F_{1}\left(x_{i}, 0, \varphi_{i}\right), \Delta^{0}, m_{i}^{c}\right) \cap S_{2}\left(F_{2}\left(x_{i}, z, \varphi_{i}\right), \Delta^{0}, m_{i}^{c}\right) \bigcap \\
& \bigcap S_{3}\left(F_{3}\left(y_{i}, 0, \varphi_{i}\right), \Delta^{0}, m_{i}^{c}\right) \bigcap S_{4}\left(F_{4}\left(y_{i}, A, \varphi_{i}\right), \Delta^{0}, m_{i}^{c}\right)
\end{aligned}
$$

It is necessary to note that after introducing into consideration the angular parameters the analytical defining the condition (3) is getting more complex one.

1. The preimage of each generating plane $E^{l}$ in space $R^{6}$ is the surface $\gamma_{l}$ which has the analytical description in the form of system $\left\{f_{1}, f_{2}, f_{3}\right\}$ depending on the geometric characteristics of the surface $\gamma_{l}$ like

$$
\left\{\begin{aligned}
&\left(a^{l} \cos \varphi_{i}-b^{l} \sin \varphi_{i}\right)\left(x_{j}-x_{i}\right)+\left(b^{l} \cos \varphi_{i}+a^{l} \sin \varphi_{i}\right)\left(y_{j}-y_{i}\right)+ \\
& f_{1}: \quad\left(a^{l} x_{j}^{k}+B^{l} y_{j}^{k}\right) \cos \left(\varphi_{j}-\varphi_{i}\right)+\left(b^{l} x_{j}^{k}-a^{l} y_{j}^{k}\right) \sin \left(\varphi_{j}-\varphi_{i}\right)-\left(a^{l} x_{i}^{h}+b^{l} y_{i}^{h}\right) \leq 0 \\
& f_{2}: 0 \leq \varphi_{i} \leq 2 \pi \\
& f_{3}: \varphi_{i}-\hat{\varphi}_{j k}^{-} \leq \varphi_{j} \leq \varphi_{i}+\hat{\varphi}_{j k}^{+}
\end{aligned}\right.
$$

where $t_{j}^{k}=\left\{x_{j}^{k}, y_{j}^{k}\right\}, k \in\left\{1, \ldots N_{j}\right\}$ and $t_{i}^{h}=\left\{x_{i}^{h} y_{i}^{h}\right\}, h \in\left\{1, \ldots N_{j}\right\}$ are vertices of objects $T_{i}$ and $T_{j}$ respectively.

2. Using the representation of each of objects $T_{i}$ in the form $T_{i}=\bigcup_{g_{i}=1}^{G_{i}} T_{g_{i}}$, where $T_{g_{i}}$ is convex and $\bigcap_{g_{i}=1}^{G_{i}} T_{g_{i}}=\emptyset$ and taking into account that under consideration of the condition (3) for each pair of objects $T_{g_{i}}$ and $T_{g_{j}}$ coefficients $a^{l}, b^{l}, c^{l}$ are functions of arbitrary vertices of objects $T_{g_{i}}$ and $T_{g_{j}}$, we can write general structure $S_{i j}$ describing condition (3) for objects $T_{i}$ and $T_{j}$ in the following way:

$$
S_{i j}=\bigcap_{g_{i}}^{G_{i}} \bigcap_{g_{j}}^{L_{j}} S\left(F\left(v_{g_{i}}, v_{g_{j}}, \varphi_{i}, \varphi_{j}\right), m\left(\varphi_{i}, \varphi_{j}\right), \Delta^{0}\right) .
$$

Remark 1 Number $m\left(\varphi_{i}, \varphi_{j}\right)$ defining the amount of inequalities in set $F\left(u_{g_{i}}, u_{g_{j}}\right)$ is a variable value and it depends on values $\varphi_{i}$ and $\varphi_{j}$. 
It is necessary to note that:

1. Support functions $f_{c}\left(v_{i}, \varphi_{i}\right)$ are concave at each point of region $D$.

2. Functions like $f_{1}$ from (11) in general (depending on the geometric characteristics of objects) have indefinite Hessian.

3. In general feasible region $D$ is a non-connected set with a finite number $\eta$ of connected components $D_{r}$, each of which in turn admits a representation in the form of a union of a finite number $\omega$ of such a non-convex subsets $D_{r k}$, that

$$
D=\bigcup_{r}^{\eta} D_{r}=\bigcup_{r}^{\eta}\left(\bigcup_{k}^{\omega} D_{r k}\right)=\bigcup_{w=1}^{W} D_{w}
$$

4. It is follows from above properties the problem (1-3) is multiextremal.

\section{METHOD OF SOLVING BASIC PROBLEM}

The following strategy for solving the local optimization problem is proposed:

1. Having fixed all the parameters $\varphi_{i}$ (e.g. $\varphi_{i}=0$ ) so that all the problem constraints became linear ones we solve the problem of local minimization objective function $z$ on a some non-convex connected component $D_{\delta}$ of region $D_{\text {linear }} \subset R^{2 n+1}\left(D_{\delta}=\bigcup_{q=1}^{Q} D_{\delta q}, D_{\delta q}\right.$ is convex subregion). In other words we solve the problem searching for $z\left(v^{*}\right)=\min _{D_{\delta} \subset R^{2 n+1}} z$ on a structure $S_{\delta}(F(v, z), \Delta, m)$ of linear inequalities (see Stoyan, Yu.G., Novozhilova, M.V., and Kartashov, A.V., (1994)) and structure $S_{\delta}$ may be represented by formula:

$S_{\delta}(F(v, z), \Delta, m)=\bigcup_{q=1}^{Q} S_{q}\left(F^{q}(v, z), \Delta^{1},\left(m^{q}=4 n+n(n-1) / 2\right)\right)$,

where each $S_{q}\left(F^{q}(v, z), \Delta^{1}, m^{q}\right)$ is a system of linear inequalities.

The point $v^{*}$ in general is described by several systems of equations $F x=B$, which may belong to different systems $S_{q}$.

2. We choose one of the systems $S_{q}^{*}\left(F^{q}(v, z), \Delta^{1}, m^{q}\right)$ and construct the mapping of the set of linear systems $\left\{S_{q}\left(F^{q}(v, z), \Delta^{1}, m^{q}\right)\right\}$ onto a set of nonlinear systems $\left\{S_{q}^{n}\left(F^{q}(v, z)\right.\right.$, $\left.\left.\Delta^{\mathbf{1}}, m^{q}\right)\right\}$ by the following rule: each constraint of type (7) is substituted by constraint of type (15) and each constraint of type (12) is substituted by constraint of type (13). Then the system $S_{q}^{* n}\left(F^{q}(v, z), \Delta^{1}, m^{q}\right)$ will define some region $D \subset R^{3 n+1}$.

3. Considering the problem of local optimization on the system $S_{q}^{* n}\left(F^{q}(v, z), \Delta^{1}, m^{q}\right)$ as a minimax problem in the space $R^{3 n}$ with the objective function $\Re=\max _{i}\left(x_{i}+x_{i}^{c}\left(\varphi_{i}\right)\right)$, where function $\Re$ is convex, we solve the problem

$$
\begin{array}{cc} 
& \min \Re \\
\text { s.t. } & S_{q}^{*}\left(F^{q}(v, z), \Delta^{1}, m^{q}\right)
\end{array}
$$

The problem belongs to a well-known problems of non-linear programming, the method- 
ology of projected Lagrangian was selected by the authors for solving the problem. More exactly, the method of sequential quadratic programming is utilized, where the approximation of Lagrange function of problem (19) is present in the objective function of auxiliary problem. The initial active set is chosen from set of constraints, which corresponds to linear ones active at the point $v^{*}$.

It is essential that

1. Hessian of problem (19) Lagrange function may be easily calculated at each point of the region $D$ by means of obvious transformations, however it is undetermined at each point of the region $D$ that is why in finding a descent direction of a objective function the modified $L D L^{T}$ decomposition of Hessian is performed.

2. The step value is chosen not only from method convergence condition but with regard for geometric characteristics of objects being allocated, namely range of parameters $\varphi_{i}$, as well. It is necessary to note, that in defining descent direction the constraints determining the range of $\varphi_{i}$ are not taken into consideration.

\section{REFERENCES}

Beasley, J.E. (1985) An exact two-dimensional non-guillotine cutting tree search procedure. Operational Research 33, 49-65.

Dowsland, K.A. and Dowsland, W.B. (1992) Packing problems. European Journal of Operational Research, 56, 2-14.

Gil, P.E., Murray, W. and Wright, M.H. (1981) Practical Optimization. Academic Press, London, New York.

Li, Zh. and Milenkovic, V. (1993) A compaction algorithm for non-convex polygons and its application, in 9th Annual ACM Symposium on Computational Geometry, May 19-21.

Magas, S.L. (1984) Methods of Solving Extremal Problems in Allocation of Polygonal Geometric Objects on a Strip. Ph.D.Thesis, Moscow. (Russ.)

Schwartz, T.J. and Sharir, M. (1983) On the "Piano Movers" Problem. I. The case of Two-Dimensional Rigid Polygonal Body Moving Admist Polygonal Barriers. Communications on Pure and Applied Mathematics, XXXVI, 345-98.

Stoyan, Yu.G. (1980) On one Generalization of the Dense Allocation Function. Reports Ukrainian SSR Academy of Science, Ser.A. 8, 70-4. (Russ.).

Stoyan, Yu.G., Novozhilova, M.V. and Kartashov, A.V. (1994) Mathematical Model and Method of Searching for a Local Extremum for the Non-convex Oriented Polygons Allocation Problem. The manuscript has been refereed and recommended for publication in European Journal of Operational Research.

Sweeney, P.E. and Ridenour, E.L. (1989) Cutting and Packing Problems: a Categorized, Application Orientated Research Bibliography. Working Paper 610, School of Business Administration, University of Michigan.

Terno, J.R. and Scheithauer, G. (1993) Modelling of packing problems. Working Paper. 